Volume 8, No. 7, July - August 2017

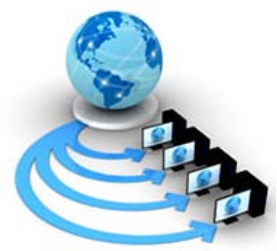

International Journal of Advanced Research in Computer Science

RESEARCH PAPER

Available Online at www.ijarcs.info

\title{
TECHNIQUES OF WIRELESS MESH NETWORKS- A REVIEW
}

\author{
Himani(PG Student), Lal Chand Panwar (Asst. professor) \\ Masters of Technology (Computer Engineering) \\ Punjabi University Patiala, Punjab, India
}

\begin{abstract}
The ad hoc network is the type of network in which mobile nodes can configure and start communicating with each other. The wireless mesh network is the type of ad hoc network in which mesh portals and mesh access points are deployed to increase scalability of ad hoc network. The wireless mesh network has decentralized architecture due to which routing, security and Quality of Service (QoS) are the three major issues in the network. In this paper, various techniques have been reviewed which had been proposed in the recent times to resolve issues of routing and security for wireless mesh networks.
\end{abstract}

KEYWORDS:Wireless mesh networks, QoS, ad-hoc.

\section{INTRODUCTION}

Wireless sensor systems (WSN), also called wireless sensor and actuator systems (WSAN), are spatially dispersed selfgoverning sensors to monitor physical or ecological conditions. The WSN is built of around thousand nodes, where every node is

connected to one (or sometimes many) sensors. Each such sensor node has commonly a few parts: a radio handset with an inner receiving wire or association with an outer reception apparatus, a microcontroller, and an electronic circuit for interfacing with the sensors and an energy source, usually a battery or an embedded form of energy harvesting

on a base station to facilitate the stream of messages to every node in the system, the individual network node forward packets to and from each other. The term "ad hoc" tends to imply "can take different forms" and "can be mobile, standalone, or networked". The detection of other nodes or devices present in the network must be detected by the ad hoc nodes. The communication and sharing of information must be allowed by the handshaking in the networks [3].

A wireless sensor network is one form of an ad hoc wireless network. A sensor network is a collection of a large number of sensor nodes that are deployed in a particular region. Sensors are wirelessly connected and they relay information back to some selected nodes. Further some computation is performed on the basis of collected data to derive an ultimate statistic to allow critical decisions to be made. There are a variety of sensors, including acoustic (sound related), seismic (an earthquake or earth vibration), image, heat, direction, smoke, and temperature sensors [4].

Vehicular adhoc network is a digital communication between two vehicles and road side buildings. It is a selforganized and distributed network. It has no fixed infrastructure so it is infrastructureless network. It works on dedicated short range communication. It can communicate between vehicle to vehicle and vehicle to roadside [5].

Wireless mesh networks (WMNs) have the potential to eliminate many of these disadvantages by offering low cost,
[1]. The cost of sensor hubs is comparatively factor, extending from a few to several rupees, contingent upon the many-sided quality of the individual sensor nodes. The resources such as energy, memory, computational speed and communication bandwidth are affected by the two factors namely size and cost. The topology of the WSNs can be either simple star network or multi-hop wireless mesh network. The routing or flooding of the hops can be seen in the propagation technique in the network [2].

There are various types of Wireless Networks. An ad-hoc network is a local area network (LAN) that is built spontaneously as devices connect. Rather than depending

wireless broadband Internet access both for fixed and mobile users. A wireless mesh network (WMN) interconnects stationary and/or mobile clients and optionally provides access to the Internet [6]. In WMN the nodes at the core of the network forwards the data to and from the clients in a multihop fashion. This forms a (mobile) ad hoc network (MANET). Many implementations allow mobile nodes to connect to the WMN. The packets in WMN are forwarded in the same multihop manner as the ones of the stationary nodes. All of the nodes present in the network do not have to support client nodes. Here the service provider can employ several relay nodes to increase the coverage of the network [7].

\section{LITERATURE REVIEW}

Yuhuai Peng, (2016) proposed in this paper [8], that network reliability is affected by the separation of control plane from the forwarding plane. In this paper, a fault tolerant routing mechanism is proposed which improves the survivability. It converts the survivability problem into two sub-problems. The one is constructing an elastic-aware routing tree and the second is the controller section. The network survivability is enhanced here on the basis of shortest path tree. This scheme continuously attempts to prune the routing tree. This fault-tolerant mechanism has proven to be better than the earlier traditional methods by 
the simulation results. The performance has been improved in terms of number of protected nodes as well as the network fragility indicator.

Prithviraj Patil, (2016) proposed in this paper [9], that the management and upgradation of the protocols is very difficult. The chances of occurrence of error are also more because the configuration must be enforced individually at each router. Software-Defined Networking (SDN) enables creating a customizable and programmable network data plane. SDN basically focused on the wired networks, for example in cloud computing. Its focus on wireless communications is less, mainly in WMNs. The limitations of SDN are presented in this paper which will further help in providing modular and flexible routing decisions along with the proper flow control. Various results are gathered which evaluate the proposed approach. The results are evaluated on the basis of QoS metrics like latency and bandwidth utilization. It is seen that the new proposed technique is more suitable for the requirements of mission-critical WMNs.

Apostolos Apostolaras, (2016) proposed in this paper [10], the design, and evaluated an information offloading architecture, where mobile users are offloaded to mesh networks, which are assembled and managed by residential users. An analytical framework is proposed that determines the offloading policy, i.e. which mobile users ought to be offloaded, based on the energy cost induced to the cellular base stations. As needed, a minimum-cost servicing policy is proposed for the mesh networks. Such architectures are feasible just if the mesh nodes agree with each other to together serve the offloaded traffic. To accomplish this, the Shapley value rule is employed for dispensing the leasing installment among the mesh nodes. The work is examined by simulating the operation of the LTEA network, and conducting testbed experiments for the mesh network. The results reveal significant savings for eNBs power consumption and reimbursements for mesh users.

Ahmed Al-Saadi, (2015) proposed in this paper [11], a heterogeneous metropolitan area network architecture that joins an IEEE 802.11 wireless mesh network with a longterm evaluation (LTE) network. Also, another heterogeneous routing protocol and a routing algorithm based on reinforcement learning called Cognitive Heterogeneous Routing (CHR) are proposed to choose the appropriate transmission technology based on parameters from every network. The simulation results demonstrate that the proposed network accomplishes an increase of up to $200 \%$ increase in throughput compared with Wi-Fi-just networks or LTE-only networks.

LI Yangfan, (2015) exhibited in this paper [12], a centralized channel assignment scheme for multi-radio multichannel wireless mesh networks. The scheme incorporates a spanner-based topology control operation and a link ranking channel assignment algorithm. The channel assignment method improves the network throughput by means of ranking links based on the principle of load balance. The simulation results demonstrate that the proposed scheme outperforms the compared centralized channel assignment approach in terms of network throughput. The test results likewise indicate that the effectiveness of the proposed scheme is even evident on account of heavy input traffic load where the potential interference in the network is severe.

Chun-Cheng Lin, (2014) proposed in this paper [13], a Router Node Placement (RNP) problem with a service priority constraint in which the mesh clients with service priorities higher than a threshold must be served. Given that this problem inherited from the complexity of the original RNP problem is computationally intractable generally, this paper develops a novel simulated annealing (SA) approach that considers momentum terms to improve the efficiency and accuracy of annealing schedules and prevent fluctuations in values of the acceptance probability function. Also, the time complexity of the proposed SA algorithm is evaluated. Besides, evaluation of various size instances under different parameters and annealing schedules demonstrate the superiority of the proposed approach.

Wanqing Tu (2015) proposed in this paper [14], how to improve performance-guaranteed multicasting transmission coverage for wireless multi-hop mesh networks by exploring the transmission opportunity offered by multiple rates (MR) and multiple channels (MC). The reliable interferencecontrolled connections and the novel MRMC multicast algorithm (LC-MRMC) are combined and a new technique is designed to make efficient utilization of channel and rate resources to significantly extend wireless multicast coverage with high throughput and short delay performance. The NS2 simulation results prove that ART and LC-MRMC accomplished improved wireless transmission quality crosswise over much bigger zones as compared to other related studies.

Jin Wang, (2015) proposed in this paper [15], a plan to explore the potential of LNC to ensure the flow untraceability and movement untraceability. In particular, first the necessary and sufficient conditions are enlisted, with which the two privacy requirements can be accomplished without encrypting either GEVs or message contents. Further, a deterministic untraceable LNC (ULNC) scheme is designed to provide flow untraceability and movement untraceability when the sufficient and necessary condition is satisfied. This method likewise gives broad theoretical examination on the probability that the condition is satisfied and additionally abundant discussions on the key parameters that affect the value of the probability. It is seen that given the low computational complexity of the proposed ULNC, this means this approach can be exceptionally promising particularly in largescale WMN.

Yuanhuizi Xu, (2015) proposed in this paper [16], dMaxQ, a novel queue length aware distributed link scheduler that requires just a single hop neighbors queue information and utilizes the celebrated max weight policy in a distributed manner. The performance of dMaxQ is evaluated and analyzed in various network topologies for both single hop and multihop traffic models, and compared it against different approaches including two queue length aware centralized algorithms, and state-of-the-art distributed approaches: JazzyMAC and ROMA. The results demonstrate that for single-hop and multi-hop traffic situations, dMaxQ acquires, separately, 100\% and 90\% of the throughput accomplished by the theoretical, centralized 
policy. Other distributed algorithms, for example,

JazzyMAC just managed $25 \%$ of the theoretical throughput.

\begin{tabular}{|c|c|c|c|}
\hline Name & ear & Description & Outcomes \\
\hline $\begin{array}{l}\text { Yuhuai Peng, } \\
\text { Xiaoxue Gong, Lei } \\
\text { Guo, Dezhi Kong }\end{array}$ & 16 & $\begin{array}{l}\text { In this paper, a fault tolerant routing } \\
\text { mechanism is proposed which } \\
\text { improves the survivability. It } \\
\text { converts the survivability problem } \\
\text { into two sub-problems. }\end{array}$ & $\begin{array}{l}\text { The performance has been improved } \\
\text { in terms of number of protected nodes } \\
\text { as well as the network fragility } \\
\text { indicator. }\end{array}$ \\
\hline $\begin{array}{lr}\text { Prithviraj } & \text { Patil, } \\
\text { Akram } & \text { Hakiri, } \\
\text { Yogesh Barve and } \\
\text { Aniruddha Gokhale }\end{array}$ & 016 & $\begin{array}{l}\text { In this paper it is proposed that the } \\
\text { management and upgradation of the } \\
\text { protocols is very difficult. The } \\
\text { chances of occurrence of error are } \\
\text { also more because the configuration } \\
\text { must be enforced individually at } \\
\text { each router. }\end{array}$ & $\begin{array}{l}\text { The results are evaluated on the basis } \\
\text { of QoS metrics like latency and } \\
\text { bandwidth utilization. It is seen that } \\
\text { the new proposed technique is more } \\
\text { suitable for the requirements of } \\
\text { mission-critical WMNs. }\end{array}$ \\
\hline $\begin{array}{l}\text { Apostolos } \\
\text { Apostolaras, George } \\
\text { Iosifidis, Kostas } \\
\text { Chounos, Thanasis } \\
\text { Korakis rand } \\
\text { Leandros Tassiulas }\end{array}$ & 016 & $\begin{array}{l}\text { An analytical framework is proposed } \\
\text { that determines the offloading } \\
\text { policy, i.e. which mobile users ought } \\
\text { to be offloaded, based on the energy } \\
\text { cost induced to the cellular base } \\
\text { stations. }\end{array}$ & $\begin{array}{l}\text { The work is examined by simulating } \\
\text { the operation of the LTEA network, } \\
\text { and conducting testbed experiments } \\
\text { for the mesh network. The results } \\
\text { reveal significant savings for eNBs } \\
\text { power consumption and } \\
\text { reimbursements for mesh users. }\end{array}$ \\
\hline $\begin{array}{l}\text { Ahmed Al-Saadi, } \\
\text { Rossitza Setchi, } \\
\text { Yulia Hicks, and } \\
\text { Stuart Allen }\end{array}$ & 2015 & $\begin{array}{l}\text { In this paper heterogeneous } \\
\text { metropolitan area network } \\
\text { architecture is proposed that joins an } \\
\text { IEEE 802.11 wireless mesh network } \\
\text { with a long-term evaluation (LTE) } \\
\text { network. }\end{array}$ & $\begin{array}{l}\text { The simulation results demonstrate } \\
\text { that the proposed network } \\
\text { accomplishes an increase of up to } \\
200 \% \text { increase in throughput } \\
\text { compared with Wi-Fi-just networks } \\
\text { or LTE-only networks. }\end{array}$ \\
\hline $\begin{array}{ll}\text { LI Yangfan, } & \text { WU } \\
\text { Peng, and } & \text { LIU } \\
\text { Xinhua } & \end{array}$ & 15 & $\begin{array}{l}\text { In this paper a centralized channel } \\
\text { assignment scheme for multi-radio } \\
\text { multichannel wireless mesh } \\
\text { networks is proposed. The scheme } \\
\text { incorporates a spanner-based } \\
\text { topology control operation and a link } \\
\text { ranking channel assignment } \\
\text { algorithm. }\end{array}$ & $\begin{array}{l}\text { The test results likewise indicate that } \\
\text { the effectiveness of the proposed } \\
\text { scheme is even evident on account of } \\
\text { heavy input traffic load where the } \\
\text { potential interference in the network } \\
\text { is severe. }\end{array}$ \\
\hline $\begin{array}{l}\text { Chun-Cheng Lin, Lei } \\
\text { Shu, and Der-Jiunn } \\
\text { Deng }\end{array}$ & 2014 & $\begin{array}{l}\text { This paper develops a novel } \\
\text { simulated annealing (SA) approach } \\
\text { that considers momentum terms to } \\
\text { improve the efficiency and accuracy } \\
\text { of annealing schedules and prevent } \\
\text { fluctuations in values of the } \\
\text { acceptance probability function. }\end{array}$ & $\begin{array}{l}\text { The evaluation of various size } \\
\text { instances under different parameters } \\
\text { and annealing schedules demonstrate } \\
\text { the superiority of the proposed } \\
\text { approach. }\end{array}$ \\
\hline Wanqi & 2015 & $\begin{array}{l}\text { This paper proposes how to improve } \\
\text { performance-guaranteed } \\
\text { multicasting transmission coverage } \\
\text { for wireless multi-hop mesh } \\
\text { networks by exploring the }\end{array}$ & $\begin{array}{l}\text { The NS2 simulation results prove that } \\
\text { ART and LC-MRMC accomplished } \\
\text { improved wireless transmission } \\
\text { quality crosswise over much bigger } \\
\text { zones as compared to other related }\end{array}$ \\
\hline
\end{tabular}




\begin{tabular}{|c|c|c|c|}
\hline & & $\begin{array}{l}\text { transmission opportunity offered by } \\
\text { multiple rates (MR) and multiple } \\
\text { channels (MC). }\end{array}$ & studies. \\
\hline $\begin{array}{l}\text { Jin Wang, Kejie Lu, } \\
\text { Jianping Wang, } \\
\text { Junda Zhu and } \\
\text { Chunming Qiao }\end{array}$ & 2015 & $\begin{array}{l}\text { In this paper a plan is proposed to } \\
\text { explore the potential of LNC to } \\
\text { ensure the flow untraceability and } \\
\text { movement untraceability. }\end{array}$ & $\begin{array}{l}\text { It is seen that given the low } \\
\text { computational complexity of the } \\
\text { proposed ULNC, this means this } \\
\text { approach can be exceptionally } \\
\text { promising particularly in largescale } \\
\text { WMN. }\end{array}$ \\
\hline $\begin{array}{lr}\text { Yuanhuizi } & \text { Xu, } \\
\text { Kwan-Wu Chin, } \\
\text { Raad Raad and } \\
\text { Sieteng Soh }\end{array}$ & 2015 & $\begin{array}{l}\text { In this paper, dMaxQ, a novel queue } \\
\text { length aware distributed link } \\
\text { scheduler is proposed that requires } \\
\text { just a single hop neighbors queue } \\
\text { information and utilizes the } \\
\text { celebrated max weight policy in a } \\
\text { distributed manner. }\end{array}$ & $\begin{array}{l}\text { The results demonstrate that for } \\
\text { single-hop and multi-hop traffic } \\
\text { situations, dMaxQ acquires, } \\
\text { separately, } 100 \% \text { and } 90 \% \text { of the } \\
\text { throughput accomplished by the } \\
\text { theoretical, centralized policy. }\end{array}$ \\
\hline
\end{tabular}

\section{CONCLUSION}

The wireless mesh network is the the decentralized and self configuring type of network in which no central controller is present due to which security, routing and quality of services come into existence. In this paper, various techniques which are applied to resolve these issues are reviewed and discussed. In future technique will be proposed for the detection of DDOS attack for wireless mesh networks.

\section{REFERENCES}

[1] B. Schrick and M. Riezenman, "Wireless broadband in a box,” 2002, IEEE Spectrum Magazine, pp. 38-43

[2] D. Beyer, "Fundamental characteristics and benefits of wireless routing ("mesh") networks," 2002, in Proc. of the International Technical Symposium of the Wireless Communications Association

[3] J. Jun, P. Peddabachagari, and M. L. Sichitiu, "Theoretical maximum throughput of IEEE 802.11 and its applications," 2003, in Proc. Second IEEE International Symposium on Network Computing and Applications (NCA 2003), (Cambridge, MA), pp. 249-256

[4] J. Jun and M. L. Sichitiu, "Fairness and QoS in multihop wireless networks," in Proc. of the IEEE Vehicular Technology Conference (VTC 2003), (Orlando, FL), Oct. 6-9 2003.

[5] K. Jain, J. Padhye, V. Padmanabhan, and L. Qiu, "Impact of interference on multi-hop wireless network performance," in Proc. of Mobicom, (San Diego, CA), 2003.

[6] J. Jun and M. L. Sichitiu, "The nominal capacity of wireless mesh networks,” 2003, IEEE Wireless Communications Magazine, Special Issue on: Merging IP and Wireless Networks
[7] R. Laufer, T. Salonidis, H. Lundgren, and P. L. Guyadec, “A cross-layer backpressure architecture for wireless multihop networks,” 2014, IEEE/ACM Transactions on Networking, vol. 22, no. 2, pp. 363-376

[8] Yuhuai Peng, Xiaoxue Gong, Lei Guo, Dezhi Kong,” A Survivability Routing Mechanism in SDN Enabled Wireless Mesh Networks: Design and Evaluation”, 2016, IEEE

[9] Prithviraj Patil, Akram Hakiri, Yogesh Barve and Aniruddha Gokhale," Enabling Software-Defined Networking for Wireless Mesh Networks in Smart Environments”, 2016 IEEE

[10] Apostolos Apostolaras, George Iosifidis, Kostas Chounos, Thanasis Korakis and Leandros Tassiulas," A Mechanism for Mobile Data Offloading to Wireless Mesh Networks", 2016, IEEE TRANSACTIONS ON WIRELESS COMMUNICATIONS

[11] Ahmed Al-Saadi, Rossitza Setchi, Yulia Hicks, and Stuart Allen,” Routing Protocol for Heterogeneous Wireless Mesh Networks", 2015, IEEE

[12] LI Yangfan, WU Peng, and LIU Xinhua,” Capacity-Based Channel Assignment Scheme in Multi-radio Multi-channel Wireless Mesh Networks", 2015, Chinese Journal of Electronics, Vol.24, No.2

[13] Chun-Cheng Lin, Lei Shu, and Der-Jiunn Deng,” Router Node Placement With Service Priority in Wireless Mesh Networks Using Simulated Annealing With Momentum Terms", 2014, IEEE

[14] Wanqing Tu,” Efficient Wireless Multimedia Multicast in Multi-rate Multi-channel Mesh Networks”, 2015, IEEE

[15] Jin Wang, Kejie Lu, Jianping Wang, Junda Zhu and Chunming Qiao,” ULNC: An Untraceable Linear Network Coding Mechanism for Mobile Devices in Wireless Mesh Networks", 2015, IEEE

[16] Yuanhuizi Xu, Kwan-Wu Chin, Raad Raad and Sieteng Soh,” A Novel Distributed Max Weight Link Scheduler for Multi-Transmit/Receive Wireless Mesh Networks”, 2015, IEEE 